LA-6124-MS

Informal Report

\section{Very High Intensity Reaction Chamber Design}

\author{
by \\ J. J. Devaney
}

UC-22 and UC-34

Reporting Date: September 1975 Issued: October 1975

An Alfirmative Action/Equal Opportunity Employer 
In the interest of prompt distribution, this report was not edited by the Technical Information staff.

For related reports see LA-5986-MS, Vols. I, II, and III.

Printed in the Uniled States of America. Available trom

National Technical Information Service

US Depariment of Commerce

5285 Port Royal Road

Springtiold, VA 22151

Price: Printed Copy \$4.00 Microtiche \$2.25

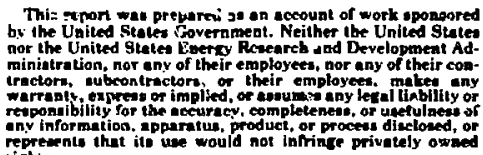

represents that ite use would not infringe privately owed

rephes. 


\section{VERY HIGH INTENSITY REACTION CHAMBER DESIGN}

by

J. J. Devaney

\section{ABSTRACT}

The problem of achleving very high intensity irradiation by light in minimal regions is studied. Three types of irradiation chamber are suggested: the common laser-reaction chamber, the folded concentric or near-concentric resonator, and the asymnetric confocal resonator. In all designs the ratio of high-intensity illuminated volume to other volume is highly dependent (to the $3 / 2$ power) on the power and fluence tolerances of optical elements, primarily mirrors. Optimization of energy coupling 1 is discussed for the common cavity. For the concentric cavities, optimization for both coherent and incoherent beams is treated. Formulae and numerical examples give the size of chambers, aspect ratios, maximum pass number, image sizes, fluences, and the like. Similarly for the asymmetric confocal chauber, formulae and numerical examples for tluences, dimensions, losees, and totally contained pass numbers are given.

\section{INTRODUCTION}

To achieve light intensities in a reaction chamber exceeding the tolerance of optical elements, It's clear that the light must be focused beyond the optical elements. For highest absolute efficiency, the light should be repeatedly refocuseci as much as losses permit, and the source-to-absorber coupling should be maximum. For the highest relative eff 1 ciency, the reaction volume exclusively should be strongly and completely illuminated. When absorber depletion is important, the refocusing should be on new aosorber material. Accordingly, our objectives are:

1. Maximum source-to-absorber coupling,

2. Perlodic refocusing of the light beam,

3. Minimum superfluous volume (1.e., least unilluminaced or weakly flluminated volume), and

4. Optimization of reaction-illuminated volume and beam intensity to prevent jverdeplation of reactants.

For really high intensities one is naturaliy drawn to laser sources and this report, therefore, considers only those. For lesser intensities, particularly when the intensities needed are comparable to or less than mirror tolerances, the designs of this report are not optimum; for such intensitfes see rather Vols. I, II, and III. 1,2,3

We suggest three chamber designs to achieve our ends: a common laser-reactor cavity, in Sec. II; folded near-concentric and concentric resonators in Sec. III; and the asymmetric confocal resonator in Sec. IV. In the common cavity design the reaction chamber is part of the laser resonator. Partial or full decoupling of laser and reaction chamber is also possible. In the fully coupled common cavity every pass of light through the laser is focused in the reaction chamber, thus utflizing the higher circulating power of the laser itself. Moreover, the efficlency of coupling the laser light to the cavity approaches $100 \%$. However, if the reaction chamber absorption is appreciable, optimum light intensities will be attained by decoupling the lasing frow the reacting medium. The folded near-concentric resonator and the asymetric confocal resonator use external laser beams. The comon and the folded resonators refocus on every pass, the confocal resonator on every other pass, but in addition compresses 
the beam itself to possibly useful intensities. Both the common and the confocal refocus to the same region, whereas the concentric refocuses at a different place along a Lissajous figura, one limiting form of which could be just two points. Each design has advantages and disadvantages which we discuss in detall below.

\section{COMMON CAVITY}

As shown in Fig. 1., the reaction chamber is an Intrinsic part of the laser resonator and forms, together with it, a stable resonator. From left to right the resonator consists of a regular fully reflecting laser end mirror $M_{1}$; the lasing medium $A$ (e.g., $\mathrm{CO}_{2}+\mathrm{N}_{2}$ gas); an optional partially reflecting mirror, either $M_{2}$ or $M_{2}^{\prime}$; a converging leng $L$, which may also serve as the reaction chamber entrance window, of diameter equal to that of the desired laser high-intensity mode; an optional partially reflecting mirror and/or optlonal window $M_{2}^{\prime}$; two conical frusta apex to apex with an apex opening equal to the effective spor size, bounded by the lers $L$, and by the final laser-cavity mirror $M_{3}$. The reaction-chamber walls are formed by elther the lens $L$ or the partial mirror/window $M_{2}^{\prime}$, the conical frusta and the mirror $M_{3}$. For highest specific reactivity in the chamber, mirror (window) $M_{2}^{t}$, if used, and mirror $M_{3}$ are of the minimum size that optical tolerance will permit. The purpose of the partially reflecting mirrors $M_{2}$ or $M_{2}^{\prime}$ is to manipulate the effective (lasing) $Q$ of the smaller resonator consisting of $M_{1}, A, M_{2}$, Mirror $M_{2}\left(M_{2}^{\prime}\right)$ may be needed if chamber absorption is large to obtain lasing at the desired frequency. The mirror $M_{2}\left(M_{2}^{\prime}\right)$ may be dispensed with and control achieved with the plano-convex lens/window $L$ used either with partial silvering, no coating, or ant 1 reflection coatings as alternative methods of manipuiating the lasing Q.

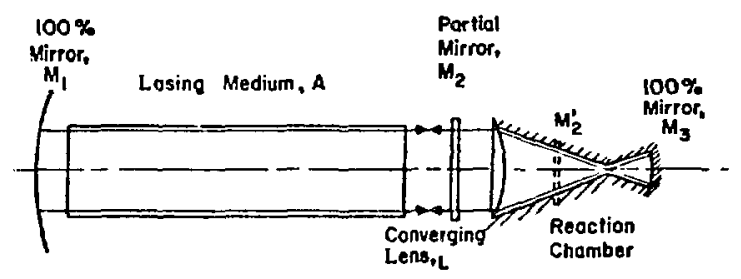

Fig. 1. High-intensity common reaction chamber.
If the losses in the reaction part of the resonator are small compared to the net galn in the laser part, the mirror $M_{2}$ (or $M_{2}^{\prime}$ ) is not needed, that is, the transmission of $M_{2}\left(M_{2}^{\prime}\right)$ and $L$ should be as close to unity as possible in order to get the maximum ifght into the reaction chamber, 1.e., to attain maximum coupling. On the other hand, for large absorption per pass in the reaction part, one wants to decouple the reaction part to a large extent, In fact to decouple the lasing part so as to make it effectively a complete laser, using regular laser techniques for maximlzing the output and neglecting the small chamber return. For intermedlate cases when the reaction-chamber net absorption is nelther large nor negligible one needs to know what reflection in $\mathrm{M}_{2}$ or $\mathrm{M}_{2}$ is opt imum. The problem is not simply the determination of the transmission $T$ of the mirror $M_{2}$ (or $M_{2}^{\prime}$ or $L$ ) for now the chamber return beam can itself Induce lasing, and several timelike modes can propagate in the system to the detrlment of the maximum intensity obtained from a single mode. The reaction-chamber return beam must therefore match in time and phase with the reflected beam. Moreover, one must be careful that absurption does not "pull" the lasing to an undestred frequency.

For lllustration we choose the simplest case. that of a steady-state laser and make the laser part and the reaction part have the same optical length measured from the mirror $M_{2}$ (or $M_{2}^{\prime}$, or L). Let the Initial gain of the laser be $\delta_{\text {mo }}$ per round trip (2 passes), the laser saturation energy be $E_{\text {sat }}$ and the iaser energy be $E_{\ell}$ so that the laser gain per round trip, $\delta$, is given by: 4

$$
\delta_{\mathrm{m}}=\delta_{\mathrm{mo}} /\left(1+E_{\ell} / E_{\mathrm{sat}}\right)
$$

Let the intrinslc fractional loss of the laser part be $\delta_{o}$ fer round trip and that of the reaction chamber be $5_{r}$ per round trip with a window or mirror transmission of $T$. of course $0 \leq T \leq 1$. (Hindow losses are included in $\delta_{r}$ or $j_{j}$ or both, as appropriate.) If $E_{r}$ is the energy in the reaction part of the resonator, then the energy increments per round trip are:

$$
\Delta \mathrm{E}_{Q}=\left[\delta_{\mathrm{m}}-\left(\bar{\delta}_{0}+\mathrm{T}\right)\right] \mathrm{E}_{\ell}+\mathrm{TE}
$$


for the laser part, and

$$
\Delta E_{r}=-\left(\delta_{r}+T\right) E_{r}+T E_{\ell}
$$

for the reaction part.

For the steady state the net energy flow 18 nil so $\Delta E_{\ell}=0=\Delta E_{I}$ and we get the relations:

$$
\left[\delta_{\mathrm{m}}-\left(\delta_{\mathrm{o}}+\mathrm{T}\right)\right] \mathrm{E}_{\ell}+\mathrm{TE} \mathrm{r}_{\mathbf{r}}=0,
$$

and

$$
\left(\delta_{r}+T\right) E_{r}=T E_{\ell}
$$

Suhstituting Eqs. (1) and (5) into (4) and solving for $E_{\ell}$ we get:

$$
\mathrm{E}_{\ell}=\left[\left(\delta_{\mathrm{mo}} / \mathrm{D}\right)-1\right] \mathrm{E}_{\mathrm{sat}}
$$

where

$$
D \equiv \delta_{0}+T-\left[T^{2} /\left(\delta_{T}+T\right]\right.
$$

For lasing we must have $E_{\ell}>0$ or

$$
\delta_{\text {mo }}>\mathrm{D}=\delta_{0}+\mathrm{T}-\left[\mathrm{T}^{2} /\left(\delta_{\mathrm{r}}+\mathrm{T}\right)\right] \text {, }
$$

which is, for our case, the usual lasing condition that the round trip gain exceed the logs. Unleos condition (8) is satisfied the common cavity will fall to lase ai all. Equation (6) agrees with Eq. (3) of Siegman ${ }^{4}$ In the limits $T=0$, or for $T=1$ and $\delta_{r} \rightarrow \delta_{e}$ Equations (5) and (6) give:

$E_{r}=\left[\frac{\delta_{\text {mo }}}{\left(\delta_{D} \delta_{\tau} / T\right)+\delta_{0}+\varepsilon_{r}}-\frac{1}{\left(\delta_{r} / T\right)+1}\right] E_{\text {sat }}$,

which we want to gaximize sulject to this condition $0<T \leq 1$. Since wa usually will have already reduced the loss parameters $\delta_{0}$ and $\delta_{r}$ tis their minlmum and the gain parameter $\delta_{\text {mo }} 0$ i:s maximum, the remaining parameter at our disposal is $T$, so we maximize Eq. (9) with respect to $\mathrm{T}$ by sitting $\partial \mathrm{E}_{\mathbf{r}} / \partial \mathrm{T}$ $=0$ and rearrange to get:

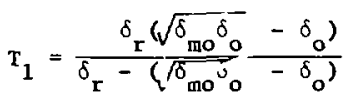

for $0<\mathrm{T}<1$ only.
The transmission, $T_{1}$, 18 that value of $T$ which maximizes the energy $E_{r}$ in the reaction chamber for the given parameters. The range of usefulness of Eq. (10) is limited and one should generally refer to Eq. (9). 'I'hus, for example, in the opt imum case that $\delta_{\text {mo }} \gg \delta_{0}, \delta_{r} ; T_{1}$ is negative and Eq. (10) does not apply. One sets $T=1$ and Eq. (9) gives the maximum reaction chamber energy, $E_{r}$. In general when $T_{1}$ of $\mathrm{Eq}$. (10) is negative the maximum $E_{r}$ from Eq. (9) 1 s obtained by putting $T=1$. A case when Eq. (10) Is useful in determining the optimum transmission occurs when the absorption in the reaction chamber is large, then

$\mathrm{T}_{1} 2 \sqrt{\delta_{\mathrm{mo}} \delta_{0}}-\delta_{0}$ for $\delta_{\mathrm{r}} \gg\left(\sqrt{\delta_{\text {iा }} \delta_{0}}-\delta_{0}\right)$,

and since $I \geq \delta_{T}$, then $T_{1} \ll 1$, and we have a highly decoupled laser. That 1s, the laser is automatically lpolaced fron the highly absorbing chamber which otherwise would quench lasing if $T$ were large. Assuming the laser lases in a single coherent mode of radius $\omega$, parallel to the optical axis, the focal fluence per pass, $n$, is given by $(R \in f, 4, p$. 316):

$$
I_{0}=E_{n} / \pi \omega_{0}^{2}=\pi E_{n} \omega^{2} / \epsilon^{2} \lambda^{2},
$$

where $E_{n}$ is the pass beam energy (clrculating laser power for $T=1$, or equals $E_{r}$ otherwise), $\omega_{0}$ is the focused spot radius; $f$ is the focal length of lens $L$; and $\lambda$ is the wavelength. If the lasing medium does not quench for a time $\tau$, the optical path length of the whole common cavity is $d$, the cavities are nearly fully coupled, and the average circulating laser energy is $E_{A}$, then the total average fluence delivered at the focus is:

$$
I_{\text {tot }}=\pi E_{A} \omega^{2} v_{c} \tau / f^{2} \lambda^{2} d=\pi E_{A} \omega^{2} v^{2} \tau / f^{2} v_{c} d,
$$

where $V_{c}$ is the velocity of light and $v$ the frequency. The total number of passes is $n=V_{c} \tau / d$. For the laser we want to maximlze the product $E_{A} \omega^{2} \tau$ for maximum fluence $I$ out; that $1 \mathrm{~s}$, we want to have a broad beam of high average energy lasting for a long time. The other parameters are the well-known geometric factors of short focal lengrh, high frequency or short wavele'tgth, and sist optical path. 
The apparent advantages of the common-cavity design are:

1. Use of the (higher) circulating laser power rather than the external beam power. For weak absorbers, a higher cavity $Q$ is possible than for external beam operacton.

2. Maximum output coupling. Laser and absorber form a comon sable resonator.

3. Very small useless reaction medium volume.

4. Very high light intensities.

5. A high number of passes, e.g., for say about $1.3 \mathrm{~m}$ cotal chamber length, $M_{1}$ to $M_{3}$ and nonquench for 200 ns one gets 46 passes through the focal point.

With disadvantages:

1. Refocus is at the same place which may deplete or bleach the reacting medium. However, one can compensate by exciting multiodes in the laser (or Increase lasing gain) and thereby enlarge the active focal reaction region.

2. If the coupiling is tight $(T \sim 1)$, absorption chamber changes affect laser operation, requiring careful tuning.

3. For maximum production we have show double duty elements such as a window performing also as a partial mirror. Effictency is galned at the cost of some complexity.

4. Early medium quench Implies ineffictent use of the available light. External cavities are then indicated.

III. FOLDED NEAR-CONCENTRIC AND CONCENTRIC RESONATORS General

The designs discussed in Secs. II and IV refocus either in precisely the same region (II) or neariy so (IV); we now suggest a design that rerocuses in different reglons in space, Indeed disparate regions if desired. We use a stable optical resonator for pertodic refocusing and introduce the beam off-aris, forming thereby a folded optical delay IIne. 5,6 our requirement that the 11 ght be refocused into a small spot on each pass forces the delay line resonator to be near concentric. For example, a confocal resonator would refocus on every other pass, permitring useless absorption in the nonfocused pass and leading to excess superflusus volume In the reaction chamber. Of course the confacal design (and others) could is used for weaker focusing on every pass with coherent beass; In ou- theory this corresponds to $\delta$ not small compared to $d$. For coherent beams our resonator will be just short of concentric; for poor beams there are advantages to the precisely concentric chamber.

Refer to Fig. 2. The react ion chamber is formed by two squashed pie segments of triangular cross section in elevation, buunded by spherical and mirrors, $M_{1}$ and $M_{2}$, spaced $d$ apart, which are of rectangular-like cross section. The mode-matching Insertion lens, L, may either be external to the chamber proper or may actually form the entrance window. Generally mirrors withstand higher beam Intensities than lenses or windows so the entrance window or lens $\&$ will, therefore, usually be further than $d / 2$ frow the line of focl. For a given spot size the minimum useless volume is achleved for d made as small as the damage threshold of the mirrore w11 permit. The foct lie along a straight line perpendicular to the optic axis in the $x-z$ plane. The origin is located where oirror $M_{1}$ extended intersects the infelal beam center, with $x$ in the long mirror dimension and $y$ in the short. The 2axis is in the Inttial cross-chamber dimension. The beam is of diameter $2 \omega$, and for a diffraction-1inited beam enters the chamber through a lens of diameter greater than $2 \omega$. Usual practice is to have an

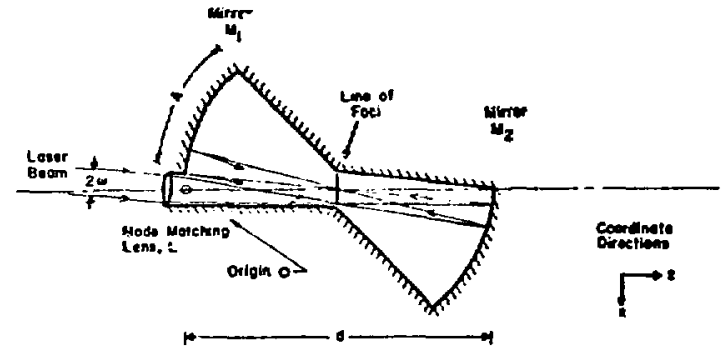

2. Tos no.

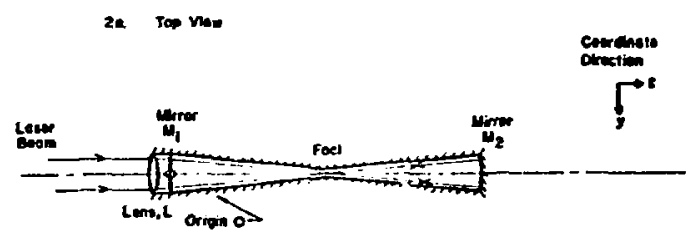

20. Sidn vien

F1g. 2. Near-concentric spherical resonator renction chamber. 
aperture 50\% greater than bean width in order to avold severe truncation and diffraction. For poorquality beams the chamber apercure actually should approach the width of the beam. For high-quality beams wider apertures might be Indicated, but not much wider, as our arguments below indicate. For our purposes the diameter 2,1 is taken as simply the destred beam truncation by the mirror $M_{1}$, regardless of actual bearn size. This parameter is a user option, but it is a critical parameter controlling pass number in a chamber of given geometry. The smaller $2 w$, the more passes can be packed 1nto the chamber. So it is, therefore, usually best to allow $2 \omega$ to ba close to the effective beam diameter. Indeed near-superradiant lasers typlcally have a high-intensity core beam with weak wings, (1.e., sort of hat shaped). However if $2 \omega$ is made too smal1, the beam is truncated and diffracted excessively. One should not in any case allow the lens $L$ to be an aperture stop of the system but rather make the mirror $M_{1}$ the 1 imiting aperture. Mirror $M_{1}$ is at the minimur distance w from the beam center at one point only, thus introducing a minimum of diffrac$t$ ton and that in the $x-z$ plane. Thus, for many beams $2 w$ can actually be the beam diameter for optimum chamber illumination. The mirrors $M_{1}$ and $M_{2}$ are spherical, of focal length $f$, and the dimension $A$ gives theit effertive arc length beginning at a distance, from the optic axis (see Fig. 3). our objectives ure to refocus the beam on each pass and to ilosely pack as many refocused images into the chamber as space will allow. To meet the formet objective requires a concentric or nearconcentric resonator. For both we ut1lize the theory of Herrlote et al. on folded optical delay lines. 5,6 We w111 first discuss the theory appropriate to coherent or neatly so heams: the treatment for poor-quality beans follows as a limit to the coherent case.

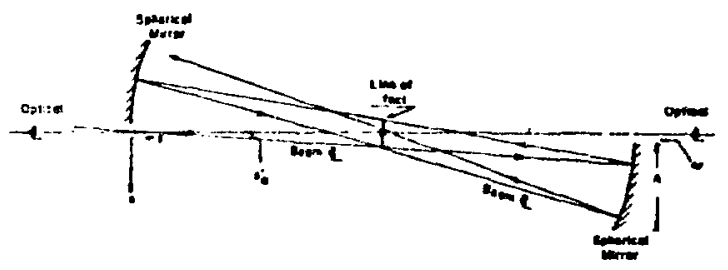

Fig. 3. Near-concentric cavity.

\section{Coherent or Diffraction-Limited Beams}

We set

$$
d+\hat{o}=4 f \text {, with } 0<\delta \ll d \text { or } f \text {, }
$$

thus forming a stable resonator. The mirror radius gat1sf 1es

$$
\mathbf{R}=\mathbf{2 f} \text {. }
$$

The usual coherent Gaussian heam-waist relation gives the focused spot radius $w_{0}: ?$

$$
\omega_{0}^{2}=\frac{\lambda}{2 \pi} \sqrt{d(2 R-d)}=\frac{\lambda d}{2 \pi} \sqrt{\frac{\delta}{d}} \text {, }
$$

which is to be compared with the spot radius from a coherent (parallel) beam of radius $b$, focused by a lens of focal length $f_{1}$, to a minimus radius $\omega_{c}$ (Ref. 4, p. 316):

$$
\omega_{0} \approx f_{1} i / \pi_{\omega} .
$$

The spot size required (and wavelength) f $1 x$ the product $d \sqrt{\delta / d}$. The corresponding beam radius is at the entrance and on the mirrors must be:

$$
L^{2}=\frac{\lambda R}{\pi} / \sqrt{2 \frac{R}{d}-1}=\frac{\lambda d}{2 \pi}\left(1+\frac{\varepsilon}{d}\right) / \sqrt{\frac{\delta}{d}} .
$$

thus ,

$$
=1 \omega \approx \sqrt{\delta / d} \text {. }
$$

Note that the power tolerances of mirrors, windows, and lenses 11mit the smallness of w.

So much for beam resonation, now for insertion. The insertion lens should match the curvature of the incoming 11ght-phase front to the curvature of the resonator wave front, 1.e., to the radius of mirror curvature $\mathrm{R}$, so that for a distant source or a parallel beam, $f_{I}=R=2 f$, where $f_{1}$ is the focal length of the ingertion lens if located at the entranct aperture. The intersection of the mirrors with the optic axis defines the origin of our Cartesian coordinate system whose $x, y$ dimensions are in a plane perpendicular to the optic axis (see Fig. 3). Let the (central) entrance beam postition be at some $x_{0}, y_{0}$, with slope $x_{0}^{\prime}\left(x_{0}^{1} \equiv d x_{0} / d z\right)$ which ts the slope of the beam center relative to the chamher 
opt ic axis, $z$. Take $y_{0}^{\prime} \equiv 0$, whlch is no loss of generallty. Each succeeding reflection on the mirrors will have successive integer Indices so that $x_{1}, y_{1}$ is the position of the beam center at the first reflection on mirror $M_{2}$, etc. Then from Herriote, Kogelnlk, and Kampfer ${ }^{5}$ we have for the successlue beam positions:

$$
x_{n}=A \sin (n \theta+a), y_{n}=B \sin (n \theta+B),
$$

where $\mathrm{n}$ is the pass number. In general, therefore, the repeated reflectlons fall on an ellipse which may In fact be converted to any Lissajous figure by making one mirtor slightly astigmatic or by adding a small beam-slzed deflecting wirror. ${ }^{6}$ Both for slaplictcy of reaction chamber design and for the gain in specific high-intensity reactivity obtainable from having the team overlap itself, we will squash the ellipse of Eq. (18) Into a straight ine and thus consider a reaction chamber of the form of FIg. 2. Following Ref. 5 and Eq. (12) we have:

$$
\cos \theta=1-(d / 2 f)=-1+(j / 2 E)
$$

and -asing $5 \ll 2 f$ :

$$
\theta \approx \pi-2 \sqrt{\delta / d}
$$

so thit for $j \ll d$, the beam cross:s to nearly the opposice, or $180^{\circ}$, position on a mirror at each pass. From Ref. 5 and Eq. (12), $A$ and $a$ are given by:

$$
\begin{array}{r}
A^{2}=(1+d / \delta)\left(x_{0}^{2}+d x_{0} x_{0}^{\prime}+d f x_{0}^{\prime 2}\right), \\
\tan 1=\sqrt{5 / d} /\left[1+2 f\left(x_{0}^{\prime} / x_{0}\right)\right]
\end{array}
$$

similarly for $a$ and $B$. However, as we noted above, we will squash the ellipse to a line (in the $x$ direction) by choosing $y_{0}, y_{0}^{\prime}=0$ so that we put $s=$ 0 .

For Itradtation of maximuw Intensity we want to pack as many passes into the chamber as possible without prematur: beam leakage through the entrance. Accordingly we calculate the spacing $\Delta_{2} x_{n}$ between reflection spcts in a given mirror:

$$
\Delta_{2} x_{n}=x_{n+2}-x_{n}=2 A \cos [(n+1) \theta+a\} \sin \theta
$$

$$
\Delta_{2} x_{n} z^{2}(-1)^{a+1} 4 A \sqrt{\delta / d} \cos [-1 d+2 \sqrt{\delta / d}(n+1)],
$$$$
\hat{0} \ll d,
$$

using Eqs. (18) and (20).

In order to just olss the entrance aperture we must spread the beas paths (1.e., make $\left.\lambda_{2} x_{0}, 0\right)$ hut as little as pussible. As discussed above we define our aperture to be $2 \omega$ and so we set

$$
2 w=\Delta_{2} x_{0}
$$

Again as discussed above, for a high-quility beam one wight want the aperture greater than the beat diameter, but usually not much mure, for beet absorption. We also want co insert the beand as such a phase $a$ in Eq. (18) where the spaclng $\therefore_{2} x_{0}$ is a relative maximum so that the rest of the bear spacings are correspondingly as close as posstale. Clearly. irom Eq. (23), that opt imum occurs in $\varepsilon_{2} x_{0}$ for $:=0$, $(\delta \ll d), 1 . e$. .

$$
\Delta_{2} x_{n} \approx(-1)^{n+1} 4 \sqrt{5 / d} \cos [2 \sqrt{5 / d}(n+1)] .
$$

$$
\therefore 2 x_{0} i-4 A \sqrt{6} / d
$$

We thus acitleve grodually increasing beam overlap elgewhere which is complete at the rurning point, pass number $n+1=(\pi / 4) \sqrt{d / \delta}$. Achieving both above objectives results in maximum passes in the arallable geometry.

Thus we inserk our beats at $x_{c}=0=y_{0}=y_{0}^{\prime}$ with $a=0$ so that $x_{0}^{\prime} \neq 0$ and

$A^{2}=(1+d / \delta) d f x_{0}^{\prime 2}=\left(d^{2} / 4\right)\left(1+\frac{d}{\delta}\right)\left(1+\frac{j}{d}\right) x_{0}^{\prime 2}$,

or

$$
A=\left(d x_{0}^{0} / 2\right)(\sqrt{d} / \delta+\sqrt{\delta / d}) .
$$

Equations (24) and (25) then give, for the insertion slope, $x_{0}^{\prime}$ of beam center :

$$
x_{0}^{\prime}=w / d,
$$

which is hardly turprising, for Eq. (27) eimply says that to move $2 \omega$ in the $x$ coordinate whlle coving $2 d$ 
In the $z$ coordinate, we get an average slope $\mathrm{dx} / \mathrm{dz}=$ $2 \mathrm{w} / 2 d$. Equation (27) fixes the Insertion slope $x_{0}^{\prime}$ in terms of chamber and beam parameters.

Refering to Eq. (26), the remaining parameter to be fixed $i s \delta$, or $\delta / d$, and $1 t$ determines or $1 s$ determined by the extent, $A$, of the beam reflection position's travel on the mirrors. To maximize the number of refocussed passes, $f_{1}$ will equal the arc length of mirror avallable, and thus, by Eq. (26), will $f 1 x: 5$. We now can compute our beam center reflection positions from Eqs. (18), (20), and (26) to be:

$$
x_{n}=(-1)^{n+1} A \sin 2 n \sqrt{\delta / d}
$$

and

$$
x_{n}=(-1)^{n+1}(\omega / 2) \sqrt{d / \delta} \sin 2 n \sqrt{\delta / d}, \delta \ll d .
$$

In our conf Iguration the beam has ef fectively leaked out of the entrance aperture when $x_{\bar{n}}=x_{0}$, giving an effective maximum pass number $\bar{n}$ from Eqs. (23), (27), and (26):

$$
2 \bar{n} \sqrt{5 / \bar{a}}=+ \text { or } \bar{n}=(\pi / 2) \sqrt{d / \delta}=\pi A / \omega \text {. }
$$

Althughi $n$ in Eq. ( $2 \delta)$ is an integer, $\bar{n}$ is not necessiarily an inceger. Noninteger values of $\bar{n}$ simply describe the situation when a urtion of the beam completes two extra passes wore thin the rest, so $\bar{n}$ is a useful ef ective pass number. We observe that $\bar{n}$ is a maximum for the largest mirror, $A$, and the smallest beam radlus or entrance aperture radius,

Equation (29) gives the maxisum effective pass number for one-half a squashed elilpse. If we let $y_{0}=\therefore$, then the return elliptic path will just mise the entrance and we can get a full ellipse. However, for a given mirror size this corresponds to central beam insertion with swings of only one-hnlf the amplitude $A$ which by E.4. (26) means $\sqrt{\mathrm{d} / \delta}+1 / 2$ $\sqrt{d / i}$ and the expected duubling of $\bar{n}$ from Eq. (29) is therefore precisely removed or compensated, so the full elilpse offers no acvantage over the half ellipse. However, there may be insertion convenlences of one versus the other.

The beam-center focused positions, $x_{f_{n}}$, may be obtained from Eq. (28) by:

$$
\begin{gathered}
x_{f_{n}}=\left(x_{n}+x_{n-1}\right) / 2 \\
=(-1)^{n+1}(\omega / 2) \cos [(2 n-1) \sqrt{\delta / d}]
\end{gathered}
$$

Where $n$ is the nth focus.

Thus the line of foci in PIgs. 2 and 3 have length $\omega+2 \omega_{0}$. From Eq. (30) we observe that as $n$ Increases from the value 1 , the focused beams overlap wore and more until at $(2 n-1) \sqrt{\delta / d}=\pi / 2$ or $x_{f_{\mathfrak{n}}}=0$, the overlap is complete; then the overlap again decreases with higher $n$. For some purposes it may be iesired to have concentrated fo: 1 or to have no mirror beam overlap. Our theory also describes such a situation. Totally disparate images may also be obtained by describing a circle in place of a squashed ellipse, or increasing $w$ and using an ellipse. The treatment is identical to the foregoing except that $x_{0}, y_{0}, y_{n}$, and $B=\alpha-\pi / 2$ are no longer zero. For the case of a circle:

$$
x_{n} \tilde{z}(-1)^{n} x_{0} \cos [(2 n-1) \sqrt{\delta / d}] \text { (circle), }
$$

and

$$
\begin{gathered}
y_{n} \approx(-1)^{n+1} x_{0} \sin [(2 n-1) \sqrt{\delta / d}], \\
\delta \ll d,(\text { circle }) .
\end{gathered}
$$

Note that we need mirrors of diameter $2\left(x_{0}+\omega\right)$ and that we describe a circle of radius $x_{0}$ on the mirrors. The foci are everily spaced around a circle of radius

$$
r=x_{0} \sqrt{\delta / d}(1+\delta / d)
$$

and are disparate when

$$
x_{0}(1+\delta / d) \sqrt{\delta / d}>\lambda d / 2 \pi \text {, }
$$

or roughly for $x_{0}>\omega$, a condition usually met or meerable in fract:ce. The maximum pass number obtained from full travel around the circle is given by

$$
\bar{n}=\pi \sqrt{d} / \delta \quad \text { (circle) }
$$

just twice ilalf an ellipse [see Eq. (29)] as it should be (but the mirrors are twice the linear 
extent and much bigger in area). Beyond these sumary remarks and Fig. 4, we say no more on the circular cavity. Unless disparate images are desired, the complexity of the chamber deplcted in Fig. 4 argues againgt its use.

\section{Coherent Beam - Concentrated Foc1}

In Figs. 2 and 3 let us replace the mirror lengths $A$ by $L$ aud reserve $A$ to be solely the amplitude of beam excursion in E'q. (28). Take the 1 imlt $A \rightarrow \infty$; then Eq. (26) Implies $\delta \rightarrow 0$, and Eq. (26) further Implies in Eq. (23) that

$$
\Delta_{2} x_{n} \rightarrow(-1)^{n+1} 2 w ;
$$

that 1s, the beam spacing along each mirror is constant with $n$ and equal to the original mirror spot diameter. The total number of passes is:

$$
\overline{\mathbf{\lambda}}=\mathrm{L} / \omega,
$$

and is quite a bit iess that half the passes iprecisely $1 / \pi$ of the pisses) obtainable through mirror overlad, Eq. (29). Equation (30) $(\delta \rightarrow 0)$ shows that the foc 1 are concentrated at the positions $x_{f}= \pm$ $w / 2$, with half the passes, namely $\bar{n} / 2=L / 2 w$. In each.

\section{Poor_Qual1ty Beams}

The foregoing theory treated a coherent beam; now suppose that the beam quality is poor, but a well-defined $f^{-} s$ of diameter $s_{1}$ is possible. All the foregoing theory of beam-center lccation, pass numbers, bean spacing, Incertion angle, and the like, remaln the same. New to this case is the lmage stze, no longer deterwined by Eq. (14) or (17), and also not necessar1ly constant. The

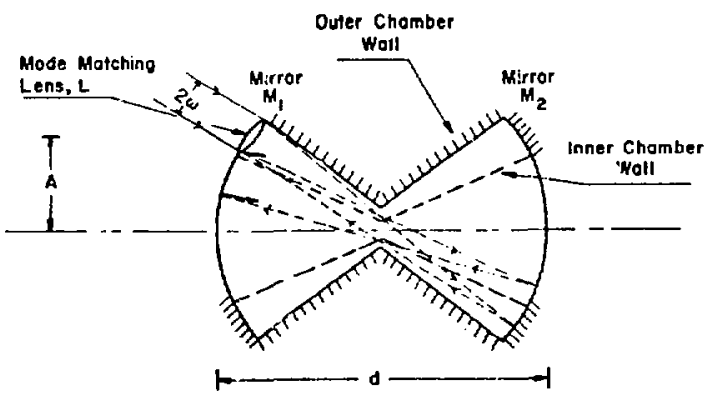

Fig. 4. Circular, near-concentrlc spherlcal-resonatri reaction chamber.
Inbertion lens provides the first focus, diameter $s_{1}$, at the center of the chamber, $Z=d / 2$. The first tmage by the mirror $M_{2}$ (Fig. 2) of $s_{1}$ is of diameter $s_{2}$. Let the object distances be $U_{n}$, the Image $U_{n}^{\prime}, n$ is the Image number, then the well-known relation

$$
i / U_{n}+1 / u_{n}^{\prime}=1 / f
$$

obtains, where $n$ is the pass number in object space. The rat to of Image size to trage distance then leads to:

$$
s_{n+1} / s_{n}=U_{n}^{\prime} / U_{n}=f /\left(U_{n}-f\right)=\frac{d-U_{n+1}}{J_{i l}} .
$$

For $\mathbf{n}=1, U_{n}=d / 2$, we then have:

$$
\mathrm{s}_{2} / \mathrm{S}_{1}=1+(2 \delta / \mathrm{d}), \delta \therefore \mathrm{d}
$$

and

$$
\left.U_{2}=(d / 2) ? 1-(2 \delta / d)\right], \delta \ll d .
$$

We must golve Eq. (38); Eq. (40) suggests that $U_{n}$ is of the form

$$
u_{n}=d / 2\left(1-\Delta_{n}\right)
$$

Substituting in Eq. (38) and using Eq. (12), we find that $\Delta$ obeys

$$
\Delta_{n+1}=\Delta_{n}+2(\delta / d) \text {. }
$$

and, from Eq. $(40), \Delta_{2}=2(\delta / d)$ so that

$$
i_{n}=2(n-1)(\delta / d) \text {, }
$$

and we have then from Eqs. (38) and (12) again:

$$
s_{n+1} / s_{n}=1+2(2 n-1)(\delta / d) .
$$

Whence for $\delta \ll d$,

$$
\begin{gathered}
s_{n} / s_{1}=\left(s_{n} / s_{n-1}\right)\left(s_{n-1} i s_{n-2}\right) \cdots\left(s_{2} / s_{1}\right), \\
s_{n} / s_{1} \approx 1+2(\delta / d) \sum_{n=1}^{n=n-1}(2 n-1) \text {, or } \\
s_{n} / s_{1} \approx 1+2 n^{2}(\delta / d), \delta \ll d ;
\end{gathered}
$$


and frow Eq. (41) and $z_{n}=v_{n}$ for $n$ even, and $z_{n}=$ $d-u_{n}$ for $n$ odd:

$$
\begin{gathered}
\left.z_{n} \approx(d / 2) \| 1+(-1)^{n+1} 2(n-1)(\delta / d)\right], \\
\delta<d
\end{gathered}
$$

Giving the relative size and location of the nth irage. Equation (4j) stowis that the trage grows quadratically in linear dinensions with pass number. We can decrease the 1.2age growth, Improve lage location control, and incruase the fasy number by making $(\delta / d)$ smali [Eq. (29)], that is, by making $A / W$ large:

$$
\sqrt{\delta / d}=\omega / 2 A \text {. }
$$

Because the mirrors are spherical we can approach total enclosure with $A \cdot \pi d / 2$ (we ignore spherical sherration for these small beams). The amount that w: can be reduced is fimfted by incensicy and hear tolerance of the mirtors.

\section{Poor Quolicy Beams - Constant Image Size}

The above theory optiolizes irradiation of a narrow linear region by maximizing the number of passes and therefore gives up constancy of the r.efocused Image size. We aiso have the option of holding the image size strictly constant by giving up a bit over $1 / 3$ the passes obtainable above. We obtain the latter case in the limit $A \rightarrow \infty$. Then Eq. (29a) showi that $5 \rightarrow 0$ and so by Eqs. (45) and (46) tha fmage sizes and positions are constant. Since Eq. (27) does not contain $A, 12$ applies as sritten and determines the insertion slope $x_{0}^{\prime}$ for the returning beam so as to just mise the entrance aperture, Horeover, Eq. (27) is predicated on an initlal 2-pass progression in the $x$ direction of one beam width, namely $2 \omega$. For $A \rightarrow \infty$ this beromes a uniform progression for all paths amcunting, therefore, to w per pass. Thus the total number of passes for mirrur length $L$ is again given by:

$\tilde{n}=$ mirror length times two/beam dtameter at entrance :onstant image size).

Table I lists a few examples of what can be dine with a poor-quality 'eam. The very large curved wrror lengrhs a (or $L$ ) chere form a nearly totally enclosed spherical Dirror cavity in the plane of the two beams, and chese carity sizes are included primarily to show the maximis absorption. Coluen 6 gives the maximus passes in the given chamber, but its hignily favorable results for near concentric (odd rows), must be tempered a bit by the results of succeeding columns which give the pass number at which the lowge linear bize has doubled (Column 7) and the central inage location at tíat pass nusber masured from the center of tha chamber (Columa 8 ). The even-numbered rows show what can be cone for constant lage size and spacing obtulned by using a spherical concentric cavity. The pass numbers for cuncentric cavities are Indeed very respectably high and therefure strict concentric is a useful option.

Systems Analys1s for the Near-Concentric Chamber = Coherent Beam Examples

Norwally in destgning an irradiation chamber, a known flued laser energy or fluence. I, is arallable and one wants to achieve a maximum focused reaction energy or fluence $I_{c}$ : thus the parameter $\omega_{0}$ is fixed by $I_{0}=I_{\omega_{0}}{ }^{2} / \omega^{2}$. Refer $=0$ Eqs. (14) and (16). The damage limitations of optical devices vill limit the beam radius $w$, and physical sizes and optics will lemis $d$ and the ratio $\mathrm{A} / \mathrm{d}$ or $\mathrm{L} / \mathrm{d}$. Thus starting with $w_{0}$ and (say) $A / d$, one gers an array of possit !e values for $w, \dot{a}, n, I$, etc., which we exhibit in Table II. However the systems analysis can be simplifted considerably by rearranging the foregoing theory. Define:

$$
a \equiv \lambda d / 2 \pi, b \equiv \sqrt{\delta / d} \text {. }
$$

Then, per Eq. (14),

$$
\omega_{0}^{2}=a b,
$$

and using Eq. (17),

$$
b=\omega_{0} / \omega \text {. }
$$

Thus ,

$$
a=w_{0} w .
$$

Equations (29) or (29a) yield 
TABLE I

POOR BEAY CONCENTRIC AND NEAR CONCENTRIC REFOCUSING EXAMPLES

$(0.5-\mathrm{cm}$ dlam laser beam focused to $0.2-\mathrm{cm}$ dirm at the center of the chamber by a $50-\mathrm{cm}$ lens)

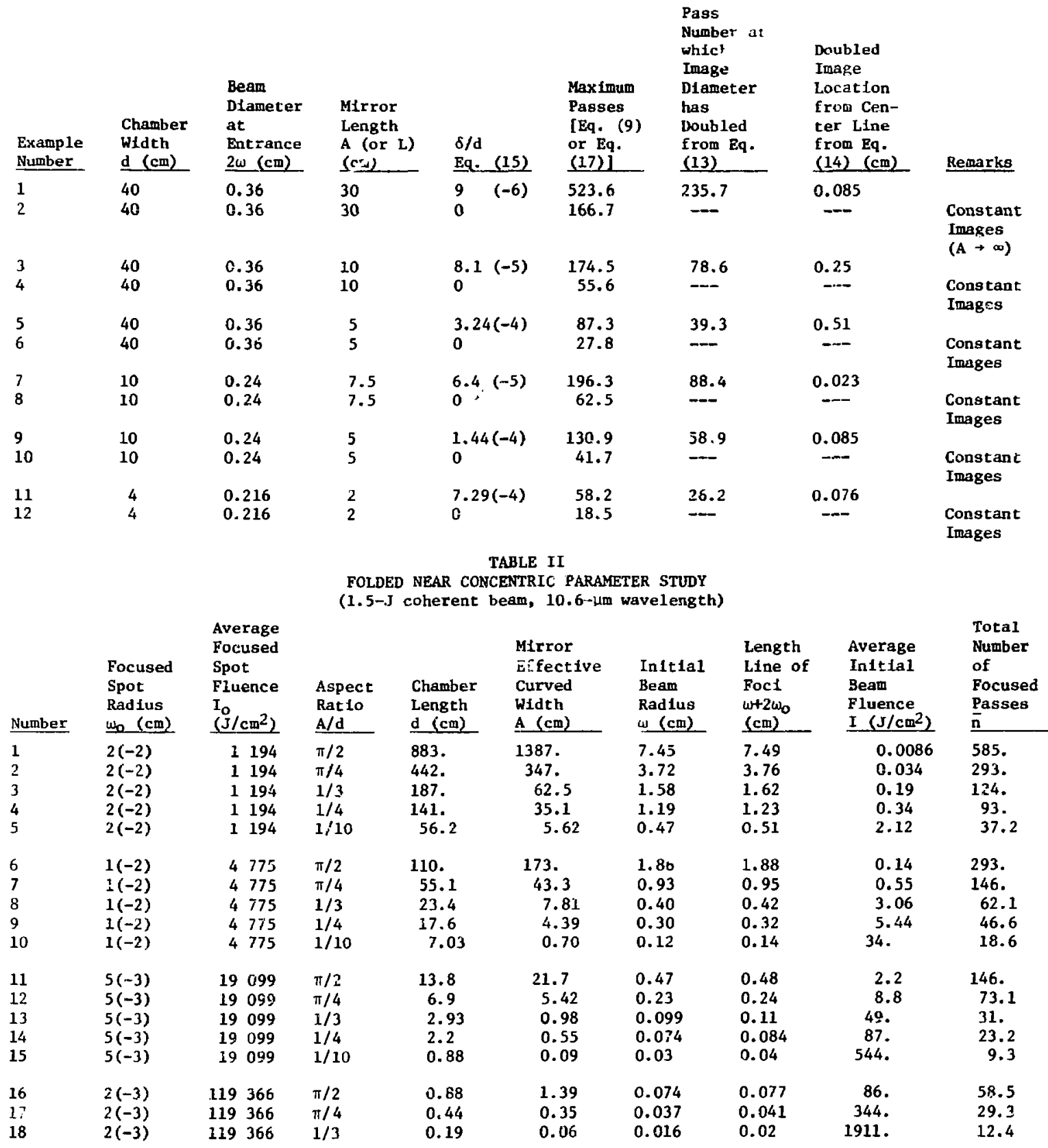




$$
A=\omega / 2 b=\omega^{2} / 2 \omega_{0}, \delta \ll d .
$$

Equation (47) then gives

$$
d=2 \pi a / \lambda=2 \pi \omega_{0} \omega / \lambda,
$$

so that

$$
A / d=(\lambda / 4 \pi)\left(\omega / \omega_{0}^{2}\right)
$$

For normal uge the aspect ratio might reasonably be $A / d \leqslant 1 / 3$, see F1g. 2. As we've noted, $A / d$ can approach total enclosure, 1.e., $\mathrm{A} / \mathrm{d} \rightarrow \pi / 2$; the 11te of foct is then surrounded by the mirrora. Much smaller aspect ratios than, say, $1 / 3$ or so gtve needlessly Ineffective absorption because of long path lengths, $d$, per pass.

Equation (29) gives the total possible number of passes before exit:

$$
\overrightarrow{\mathrm{n}}=\pi / 2 b=\pi \omega / 2 \omega_{0} .
$$

Equation (30) gives th? extent of the ilne of foc: $\omega+2 \omega_{0}$. Start1ng, therefore, with $\omega_{0}, \lambda$, and $A / d$, Eq. (53) gives $\omega$, whence Eq. (52) gives d, Eq. (54) gives $\bar{n}$, and

$$
I=E / \pi \omega^{2}
$$

gives the or 1 ginal average beam fluence at the aperture (diameter $2 \omega$ ) for beam energy $E$.

In Table II we give a study over a very limited range of parameters for a coherent 10.6- $\mu \mathrm{m}$ beam of $1.5 \mathrm{~J}$. For poor-quality beams other parameters are possible; see, for example, Tailie I. Note that Table II can be used as a basis for estimates as well as used 1iterally. Thus, for example, an intensity I of $544 \mathrm{~J} / \mathrm{cm}^{2}$ (Row 15) on the mirrors is far in excess of present day tolerances. As a second example one might use the parameters of Row 11 to get $\sim 20000 \mathrm{~J} / \mathrm{cm}^{2}$ per repeated focus from a $1-\mathrm{cm}-\mathrm{diam}-$ eter beam, but in a chamber of mirror length $L$ less than she full $\mathrm{A}=21.7 \mathrm{~cm}$ at the sacrifice of a high pass number (147). For $L<A$ the pass number 1mmediately drops to one-half or less because there is no return of the beam to the entrance.

In rable II we have chosen four focused spot sizes of radius $\omega_{0}$, and five chamber dimengiong of. differing aspect rat $10 \mathrm{~A} / \mathrm{d}$, from which, given the beam euergy and frequency, we have a completely determined problem for a coherent beam, and from which we can calculate the bean entrance aperture radius, the chamber length $d$, and the mirror length for full turnaround $A$, the intensities at beam walst and at the mirrors, and the total number of posstble passes $\bar{n}$. Mirror fluences of the order of $100 \mathrm{~J} / \mathrm{cm}^{2}$ for short ( $<1 \mu s$ ) pulses exceed tolerances at this time; such intensities are included for scallng purposes.

Advantages and DLsadvantages of the Folded Concentric and Near-Concentric Resonators

\section{Advantages}

1. Refocuses a beam to the same size, image or waist, if desired.

2. Refocuses on a line, on two points, or in general along any Liasajous figure, so that efther distributed or concentrated foct are avallable.

3. Refocuses on each pass.

4. Leaves a very sma1l, useless reaction volume in the medium.

5. Uses an exlernal beam, so that laser and reactlon chamber can be worked on separately.

6. Beam repetition can be made disparate and can thus be counted and if need be "dumped" or reinserted.

7 Images can be made disparate.

8. A high number of refocusing passes are possible, many hundreds if desired.

9. Adaptable to beams of any quality.

10. Very high 11ght intensities are possible.

\section{D1sadvantages}

1. For coherent beams the resonator and beam sizes are interdependent (Table II), limiting flexibtlity.

2. Again for coherent beams, high spot intensity implies high mirror intensities or low pass number.

3. For poor-quality beams higher pass numbers imply quadratic growth of image size.

4. Not as tightly coupled to the laser as the common cavity so that effective beam power on target is therefore lower.

5. Needs matching or insertion optics. 
IV. ASYMMETRIC CONFOCAL RESONATOR

Because the asymmetric confocal resonator ${ }^{3}$ (Fig.

5) has the property of trapping, cospressing, and focusing a beam for a time, it has merit, perhaps superfor merlt, for high-1ntensity reaction chamber design. Indeed it usually gives the highest absorption in weakly absorbing distributed media. ${ }^{3}$ However, compression and trapping of the beam are directly limited by mirror tolerances and the (strong) focusing, which is not so limited, occurs only on every other pass so that ab initio the confocal resonator is not as suttable as the foregoing designs for vexy high intensity chambers. In the notation of Raf. 3., we define the width of the cavicy between mirrors $W$, the radius of the mirrors $\ell+2 c$, the beam radius $c$, the fasertion radius $\ell+c$, and the effectlve cotal loss per rass $1-\Lambda$; and calculate thereby the parameters of Table III for an energy $E_{0}=1.5 \mathrm{~J}$ at a wavelength of $10.6 \mu \mathrm{m}$ for a coherent or diffraction-1imiced beam. The resonator will do less well for poorer beams. We take the far-field spread of the beam to be $\lambda / \pi c$ (Ref. 4, p. 309) so that,

$$
\mathrm{e} / \mathrm{d}_{\mathrm{o}}=\lambda / \pi \mathrm{c}
$$

The rat 10 of focal lengths $s$, Colum 7, Table III, is determined from Ref. 3, Eq. (42):

$(\lambda / \pi c) W s\left(s^{3}+s^{2}+s+1\right)-\hat{x} s^{2}+(\ell+2 c)=0$.

The radius of the first focal spot, $w_{0}$, Column 8 , from Ref. 3 [Eqs. (47) and (2)] is:

$$
\omega_{0}=W s \lambda / \pi c(1+s) \text {. }
$$

The average fluence at firgt focus, $I_{\text {fo }}$, Column 9 , is:

$$
I_{f o}=E_{o} / \pi \omega_{0}^{2} \text {. }
$$

The summed average fluence, I, at focus, Colum 10, 1s [Ref. 3, Eq. (50c)]:

$$
I \approx I_{\mathrm{fo}} /\left[1-\left(\Lambda^{2} / s^{2}\right)\right]
$$

The pass number, $2 n^{\prime}$, at which the beam edge just reaches the center is given by Kef. 3, Eqs. (51b)

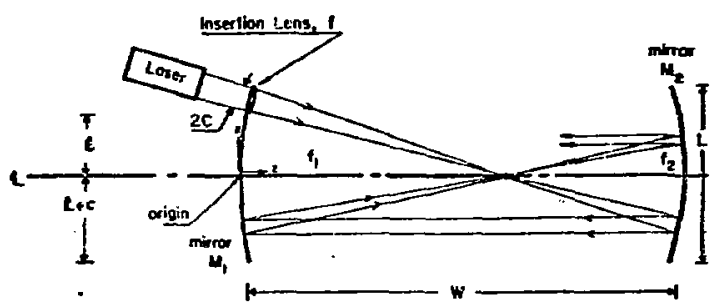

Fig. 5. Asymetric confocal resonator reaction chamber.

and $(56)$, to:

$$
2 n^{\prime}=\ln \left(\ell / \omega_{0}\right) / \ln s=\bar{n},
$$

where $2 \bar{n}$ (Column 13) is the pass number at which the beam just begins to leak bick out the entrance aperture. The cotal fluence at the mirror centers $I_{c}$, Column 11, was obtained from Eq. (54) of Ref. 3:

$$
I_{c} \approx I(\Lambda / s)^{2 n^{\prime}}
$$

The time for the beam to complete a round trip in the chamber, $T$, column 12 , Is given by

$$
\tau=2 \mathrm{~W} / \mathrm{v}_{\mathrm{c}},
$$

where $v_{c}$ is the velocity of 1ight. Last, the initial beam fluence, $I_{0}$, Column 6 , is:

$$
I_{0}=E_{0} / \pi c^{2}
$$

One figure of mertt of the confocal cavity is the ratio of the summed focus fluence, $I$, to the central mirror fluence, $I_{c}$, $f_{\text {.e. }}$, the maximum chamber $f l_{\text {ince }}$ to the maximum mirror fluence. Note that $I_{c}$ is only the maximum mirror fluence when $s / \Lambda$ is not too different from unity. From Eq. (62) and using Eqs. (61) and (58):

$$
I / I_{c}=(s / \Lambda)^{\bar{n}}=\ell \pi c(1+s) /\left(W s \lambda \Lambda^{\bar{n}}\right),
$$

so that to maxfmize this ratio we want a large mirror radius $\ell$, a large bean radius $c$, minimum focal length ratio $s>1$, minimum cross-chamber path, $W$, and maximum number of paths, $2 \bar{n}$, within the chamber [leading again to minimum $\mathrm{s}$, maximum 2 , etc. from Eq. (61)]. We do not want minimum $\Lambda$, in spite of Eq. (65), because $1-\Lambda$ is the beam absorption 
TABLE III

ASYMMETRIC, CONGOCAL RESONATOR PARAMETER STUDY (1.5-J cohcrent beam, 10.5- $\mu$ m wavelength)

\begin{tabular}{|c|c|c|c|c|c|c|c|c|c|c|c|c|}
\hline $\begin{array}{l}\text { Run } \\
\text { Num- } \\
\text { ber } \\
\end{array}$ & $\begin{array}{l}\text { Chamber } \\
\text { Length } \\
\text { W (cm) }\end{array}$ & $\begin{array}{l}\text { Chamber } \\
\text { Mirror } \\
\text { Minimum } \\
\text { Radius } \\
\ell(\mathrm{cm}) \\
\end{array}$ & $\begin{array}{l}\text { Ream } \\
\text { Rad lus } \\
\mathrm{C} \text { (cm) }\end{array}$ & $\begin{array}{l}\text { Initial } \\
\text { Beam } \\
\text { Fluence, } \\
\mathrm{I}_{\mathrm{o}} \\
\left(\mathrm{J} / \mathrm{cm}^{2}\right) \\
\end{array}$ & $\begin{array}{l}\text { Total } \\
\text { Fractional } \\
\text { Loss per } \\
\text { Pags } \\
1-\Lambda \\
\end{array}$ & $\begin{array}{l}\text { of } \\
\text { Mirror } \\
\text { Focal } \\
\text { Lengths } \\
\text { S } \\
\end{array}$ & $\begin{array}{l}\text { First } \\
\text { Focus } \\
\text { Spot } \\
\text { Radius } \\
\omega_{0} \text { (cm) }\end{array}$ & $\begin{array}{l}\text { First } \\
\text { Focus } \\
\text { Fluence, } \\
\text { I fo } \\
\left(\mathrm{J} / \mathrm{cm}^{2}\right)\end{array}$ & $\begin{array}{l}\text { Summed } \\
\text { Focus } \\
\text { Fluence, } \\
\mathrm{I} \\
\left(\mathrm{J} / \mathrm{cm}^{2}\right) \\
\end{array}$ & $\begin{array}{l}\text { Central } \\
\text { Fluence, } \\
I_{c}\left(\mathrm{~J} / \mathrm{cm}^{2}\right)\end{array}$ & $\begin{array}{l}\text { Round- } \\
\text { Trip } \\
\text { Time (2 } \\
\text { Passes) } \\
\text { (nsec) } \\
\end{array}$ & $\begin{array}{l}\text { Pass } \\
\text { Number at } \\
\text { Beginning } \\
\text { of Leak- } \\
\text { out, 2n }\end{array}$ \\
\hline $\begin{array}{l}1 \\
2 \\
2 \mathrm{~A} \\
3 \\
4 \\
4 \mathrm{~A} \\
5 \\
6 \\
7\end{array}$ & $\begin{array}{l}2 . \\
2 . \\
2 . \\
2 . \\
2 . \\
2 . \\
2 . \\
2 . \\
2 .\end{array}$ & $\begin{array}{l}0.5 \\
0.5 \\
0.5 \\
0.5 \\
0.5 \\
0.5 \\
0.5 \\
0.5 \\
0.5\end{array}$ & $\begin{array}{l}0.5 \\
0.5 \\
0.5 \\
0.2 \\
0.2 \\
0.2 \\
0.1 \\
0.1 \\
0.1\end{array}$ & $\begin{array}{l}1.91 \\
1.91 \\
1.91 \\
11.94 \\
11.94 \\
11.94 \\
47.7 \\
47.7 \\
47.7\end{array}$ & $\begin{array}{l}0.005 \\
0.10 \\
0.20 \\
0.005 \\
0.10 \\
0.20 \\
0.005 \\
0.10 \\
0.20\end{array}$ & $\begin{array}{l}1.736 \\
1.736 \\
1.736 \\
1.345 \\
1.345 \\
1.345 \\
1.186 \\
1.186 \\
1.186\end{array}$ & $\begin{array}{l}8.56(-4) \\
8.56(-4) \\
8.56(-4) \\
1.94(-3) \\
1.94(-3) \\
1.94(-3) \\
3.66(-3) \\
3.66(-3) \\
3.66(-3)\end{array}$ & $\begin{array}{rr}651 & 080 \\
651 & 080 \\
651 & 080 \\
127 & 496 \\
127 & 496 \\
127 & 496 \\
35 & 614 \\
35 & 614 \\
35 & 614\end{array}$ & $\begin{array}{rr}969 & 582 \\
890 & 380 \\
826 & 616 \\
281 & 661 \\
230 & 893 \\
197 & 309 \\
120 & 136 \\
83 & 920 \\
65 & 324\end{array}$ & $\begin{array}{c}1567 . \\
452 . \\
108 . \\
992 . \\
124 . \\
11.7 \\
762 . \\
29.6 \\
0.8\end{array}$ & $\begin{array}{l}0.13 \\
0.13 \\
0.13 \\
0.13 \\
0.13 \\
0.13 \\
0.13 \\
0.13 \\
0.13\end{array}$ & $\begin{array}{l}23 . \\
23 . \\
23 . \\
37.4 \\
37.4 \\
37.4 \\
57.6 \\
57.6 \\
57.6\end{array}$ \\
\hline $\begin{array}{l}8 \\
8 \mathrm{~A} \\
9 \\
9 \mathrm{~A} \\
10 \\
10 \mathrm{~A} \\
11 \\
12 \\
13 \\
14 \\
15 \\
16\end{array}$ & $\begin{array}{l}10 . \\
10 . \\
10 . \\
10 . \\
10 . \\
10 . \\
10 . \\
10 . \\
10 . \\
10 . \\
10 . \\
10 .\end{array}$ & $\begin{array}{l}0.5 \\
0.5 \\
2 . \\
2 . \\
2 . \\
2 . \\
2 . \\
2 . \\
2 . \\
2 . \\
2 . \\
2 .\end{array}$ & $\begin{array}{l}0.5 \\
0.5 \\
0.5 \\
0.5 \\
1 . \\
1 . \\
2 . \\
2 . \\
2 . \\
0.2 \\
0.2 \\
0.2\end{array}$ & $\begin{array}{r}1.91 \\
1.91 \\
1.91 \\
1.91 \\
0.48 \\
0.48 \\
0.12 \\
0.12 \\
0.12 \\
11.94 \\
11.94 \\
11.94\end{array}$ & $\begin{array}{l}0.005 \\
0.10 \\
0.005 \\
0.10 \\
0.005 \\
0.10 \\
0.005 \\
0.10 \\
0.20 \\
0.005 \\
0.10 \\
0.20\end{array}$ & $\begin{array}{l}1.753 \\
1.753 \\
1.226 \\
1.226 \\
1.415 \\
1.415 \\
1.733 \\
1.733 \\
1.733 \\
1.096 \\
1.096 \\
1.096\end{array}$ & $\begin{array}{l}4.30(-3) \\
4.30(-3) \\
3.72(-3) \\
3.72(-3) \\
1.98(-3) \\
1.98(-3) \\
1.07(-3) \\
1.07(-3) \\
1.07(-3) \\
8.82(-3) \\
8.82(-3) \\
8.82(-3)\end{array}$ & $\begin{array}{rr}25 & 861 \\
25 & 861 \\
34 & 573 \\
34 & 573 \\
122 & 147 \\
122 & 147 \\
417 & 175 \\
417 & 175 \\
417 & 175 \\
6 & 134 . \\
6 & 134 \\
6 & 134\end{array}$ & $\begin{array}{rr}38 & 155 \\
35 & 119 \\
101 & 384 \\
75 & 021 \\
241 & 530 \\
205 & 082 \\
622 & 219 \\
571 & 170 \\
530 & 102 \\
34 & 786 \\
18 & 809 \\
13 & 119\end{array}$ & $\begin{array}{c}314 . \\
123.6 \\
161 . \\
5.4 \\
216 . \\
24.9 \\
311 . \\
72.2 \\
13.3 \\
114.2 \\
0.2 \\
1.1(-4)\end{array}$ & $\begin{array}{l}0.67 \\
0.67 \\
0.67 \\
0.67 \\
0.67 \\
0.67 \\
0.67 \\
0.67 \\
0.67 \\
0.67 \\
0.67 \\
0.67\end{array}$ & $\begin{array}{l}17 . \\
17 . \\
61.8 \\
61.8 \\
39.8 \\
39.8 \\
27: 4 \\
27.4 \\
27.4 \\
118 . \\
118 . \\
118 .\end{array}$ \\
\hline $\begin{array}{l}17 \\
18 \\
19 \\
20 \\
21 \\
22 \\
23 \\
24 \\
25 \\
26 \\
27\end{array}$ & $\begin{array}{l}50 . \\
50 . \\
50 . \\
50 . \\
50 . \\
50 . \\
50 . \\
50 . \\
50 . \\
50 . \\
50 .\end{array}$ & $\begin{array}{l}10 . \\
10 . \\
10 . \\
10 . \\
10 . \\
10 . \\
10 . \\
10 . \\
10 . \\
10 . \\
10 .\end{array}$ & $\begin{array}{l}0.5 \\
0.5 \\
1 . \\
1 . \\
1 . \\
2 . \\
2 . \\
2 . \\
5 . \\
5 . \\
5 .\end{array}$ & $\begin{array}{l}1.91 \\
1.91 \\
0.48 \\
0.48 \\
0.48 \\
0.12 \\
0.12 \\
0.12 \\
0.02 \\
0.02 \\
0.02\end{array}$ & $\begin{array}{l}0.005 \\
0.10 \\
0.005 \\
0.10 \\
0.20 \\
0.005 \\
0.10 \\
0.20 \\
0.005 \\
0.10 \\
0.20\end{array}$ & $\begin{array}{l}1.049 \\
1.049 \\
1.096 \\
1.096 \\
1.096 \\
1.183 \\
1.183 \\
1.183 \\
1.414 \\
1.414 \\
1.414\end{array}$ & $\begin{array}{l}1.73(-2) \\
1.73(-2) \\
8.82(-3) \\
8.82(-3) \\
8.82(-3) \\
4.57(-3) \\
4.57(-3) \\
4.57(-3) \\
1.98(-3) \\
1.98(-3) \\
1.98(-3)\end{array}$ & $\begin{array}{rr}1 & 600 \\
1 & 60 C \\
6 & 137 \\
6 & 137 \\
6 & 137 \\
22 & 843 \\
22 & 843 \\
22 & 843 \\
122 & 209 \\
122 & 209 \\
122 & 209\end{array}$ & $\begin{array}{rr}15 & 958 \\
6 & 064 \\
35 & 022 \\
18 & 872 \\
13 & 147 \\
77 & 947 \\
54 & 180 \\
42 & 068 \\
241 & 942 \\
205 & 357 \\
179 & 697\end{array}$ & $\begin{array}{l}14.2 \\
8.6(-6) \\
21 . \\
5 .(-3) \\
4 .(-7) \\
28.3 \\
0.2 \\
7 .{ }^{(-4)} \\
42.3 \\
3 . \\
0.2\end{array}$ & $\begin{array}{l}3.33 \\
3.33 \\
3.33 \\
3.33 \\
3.33 \\
3.33 \\
3.33 \\
3.33 \\
3.33 \\
3.33 \\
3.33\end{array}$ & $\begin{array}{c}266 . \\
266 . \\
154 . \\
154 . \\
154 . \\
91.4 \\
91.4 \\
91.4 \\
49.2 \\
49.2 \\
49.2\end{array}$ \\
\hline
\end{tabular}


per pass and clearly we do not want to increase useless absorption. Equation (65) is only an approprlate figure of werit when the loss $(1-\Lambda)$ is small enough to make the central fluence the maxinum on the mirrors. Por small $\Lambda$, the ratio $I / I_{0}$ is a wore relevant figure of merit.

It is to be noted that the asymetric confocal resonator gatns high fluence by trapping the beam for a relatively long $t$ ime $(t=\infty$ for rays passing through the confocal point) so that time-dependent reactlons will behave differently in this chamber. Also, the image size grows proportlonal to $s^{2 n}$ (Ref. 3) where $n$ is the number of passea.

\section{Advantages of the Asymmetric Confocal Resonator}

1. Gives high volumetric, incensity-independent Irradiation efficlency, Indeed generally the highest of external beam designs.

2. Provides concentrated central 1llumination.

3. Has a small useless reaction volume in the med 1um.

4. Compresses and overlaps as well as focuses beaw.

5. Uses an external beam.

6. Yields a very high number of passed ( $\infty$ for rays through the confocal point).

Disadvantages

1. Requires high-quality beam.

2. Useless volume is the highest of the cases discussed.

3. The summed focused intensity is mirror-damage limited to a greater degree than the other designs.

4. Using an external beam means lower power compared to the common cavity.

5. Needs Insertion opt1cs.

6. Refocuses beam every other pass.

7. Image size, beam size and posicion are continually varying. Has a single maximum integrated fluence region.

\section{v. FINAL REMARKS}

It is not possible to a priori recommend one cavity over another for very high intensity 11lumination. Nor are the lists of advantages and disadvantages really suffictent for more than a first assessment. The parameters of each circumstance should be Inserted Into the approprlate formulae in order to select the best cavity. Not fully represented here 15 the 1oportant factor of conventence to the operator or experimentalist, whtch to a large extent must be individually determined.

\section{ACKWOWLEDGEMENTS}

The form of common laser-absorber cavity presented here was developed conjointly with $C$. Paul Robinson and John Lyman of the Los Alamos Sclentific Laboratory. I am Indebted to them and to Reed Jensen, Steven Rockwood, and Frederick Skoberne for critical readings of the manuscript.

\section{REFERENCES}

1. J. J. Devaney and D. E. Jackson, "OptIcal Design of a Reaction Chamber for Weakly Absorbed Light: I. Canted and Parallel Mirrors," Los Alamos Sclentiflc Laboratory report LA5986-MS, Vol. I (1975).

2. J. J. Devaney and F. T. Finch, "Optical Design of a Reaction Chamber For Weakly Absorbed Light: II. Parallel Mirrors, Mult1-Travel." Los Alamos Scientific Laboratory report LA5986-MS, Vol. II (1975).

3. J. J. Devaney, "OptJ.c.l Design of a Reaction Chamber for Weakly fabsorbed Light: III. Asymmetric Confocal Resonator," Los Alamos Scientif ic Laboratory report LA-5986-MS, Vol. III (1975).

4. A. E. Siegman, An Introduction to Lasers and Masers (McGraw-Hi11, New York, 1971), p. 432.

5. D. Herriott, H. Kogelnik, and R. Kompfer, "OffAxis Paths in Spherical Mirror Interferometers," App1. Opt. 3, 523 (1964).

6. D. R. Herrfott and H. J. Schulte, "Folded Optical Delay l.ines," Appl. Opt. 4,883 (1355).

7. H. Kogelnik and T. Li, "Laser Beams and Resonators," Appl. Opt. 5, 1550 (1966). 